\title{
Computational-Mechanism Design: A Call to Arms
}

\section{Citation}

Dash, Rajdeep K., Nicholas R. Jennings, and David C. Parkes. 2003. Computational-mechanism design: A call to arms. IEEE Intelligent Systems 18(6): 40-47.

\section{Published Version}

doi:10.1109/MIS.2003.1249168

\section{Permanent link}

http://nrs.harvard.edu/urn-3:HUL.InstRepos:4101007

\section{Terms of Use}

This article was downloaded from Harvard University's DASH repository, and is made available under the terms and conditions applicable to Other Posted Material, as set forth at http:// nrs.harvard.edu/urn-3:HUL.InstRepos:dash.current.terms-of-use\#LAA

\section{Share Your Story}

The Harvard community has made this article openly available.

Please share how this access benefits you. Submit a story.

Accessibility 


\section{A gents a n d Mar hets}

\section{Computational- Mechanism Design: A Call to Arms}

Rajdeep K. Dash and Nicholas R. Jennings, University of Southampton

David C. Parkes, Harvard University

$\mathbf{T}$ he need is increasing for computer systems that operate a decentralized control regime, are open (individual components can enter and leave at will), and contain several components representing distinct stakeholders with different aims and objectives. Relevant examples include Grid computing, the Semantic Web, pervasive

Game theory has

developed powerful tools

for analyzing decision

making in systems with

multiple autonomous

actors. These tools,

when tailored to

computational settings,

provide a foundation for

building multiagent

software systems. This

tailoring gives rise to the

field of computational-

mechanism design,

which applies economic

principles to computer

systems design. computing, e-commerce, mobile computing, and peer-to-peer (P2P) systems.

For these complex systems, agent-based approaches, which emphasize autonomous actions and flexible interactions, are natural computational models. ${ }^{1}$ In designing such multiagent systems (MASs), you must address two fundamental issues. First, you must specify the protocols that govern the interactions. These protocols cover issues such as how the agents' actions translate into an outcome, the range of actions available to the participants, and whether the interactions occur over steps or are one-shot. Second, given the prevailing protocol, you must define each agent's strategy (mapping from state history to action).

Sometimes, a designer might be able to impose each agent's protocol and strategy. In such settings, the agents can cooperate to find a good systemwide solution. ${ }^{2}$ However, usually this isn't feasible because the agents represent distinct stakeholders with potentially conflicting goals that seek to maximize their own gains. Consequently, the best a designer can achieve is a noncooperative strategic analysis, in which the designer can impose only the protocol and can't control which strategies the agents adopt. Against this background, we examine the field of computational-mechanism design. CMD provides an elegant mathematical framework in which to study protocols that give the agents incentive to act and interact in particular ways and that have useful computational properties. It is an emerging field with which MAS designers need to become familiar. Moreover, the relatively new subfield of distributedcomputational-mechanism design-which has no trusted center-is attracting considerable interest because it is a better match with the computational MAS model. However, this raises many additional open questions because the agents that must implement the mechanism's rules are those that stand to benefit from its (mis)operation.

\section{Designing MASs using mechanism design techniques}

In contrast with competitive equilibrium theory, ${ }^{3,4}$ where agents respond solely to summary signals (such as prices for different outcomes) about the multiagent problem, we assume CMD agents act in a game-theoretic way, thereby modeling the effect their actions will have on other agents' actions. This more detailed modeling facilitates the design of predictable systems of interacting agents and has caused MAS designers to start looking at game theory ${ }^{5,6}$ and, more recently, mechanism design. Specifically, MD deals with how to design systems so that certain systemwide properties (for example, efficiency, stability, and fairness) emerge in equilibrium from the constituent components' interaction. MD is particularly appealing for designing MASs with self-interested agents because it provides methods for simplifying the strategic problems facing agents at design time.

In its native form, MD is a beautiful but brittle endeavor; it models agents as rational and then optimizes with respect to this model. However, the theory's underlying assumptions can be inappropriate in computational settings because software agents are invariably bounded-rational. Also, the theory focuses on centralized mechanisms engineered so 
that agents reveal their complete private information, and the design problem is itself often intractable. Furthermore, it's assumed that communication between agents and the system is free and faultless and that the system is closed, with a static agent population. It's also assumed that agents understand the protocols that govern their interactions and abide by them. These assumptions are problematic in MASs because

- Agents don't have the unbounded computational power that might be required to calculate their preferences for all possible outcomes or calculate equilibrium strategies

- The mechanism infrastructure in a centralized mechanism might be unable to compute the outcome because the problem might be intractable

- Communication is not necessarily costfree and could also be error-prone

- Most real MASs are dynamic, and the set of agents varies with time owing to the system's open nature

- Semantic interoperation between different participants in an open system is difficult to attain, as is a machine-understandable specification of all the associated interaction protocol's aspects

CMD seeks to address these limitations and thereby apply MD techniques to computational problems. You might imagine that a new field isn't required because we can decompose the problem of using MD in a MAS into its economic part (MD) and its computational part (MAS), then attack it in a modular fashion. However, this approach fails to recognize that we must address both economic and computational principles at each stage of design. In fact, often principles from one area can help solve a problem in the other. For example, you could make finding an undesirable equilibrium strategy (an economic problem) an intractable problem (a computational solution) for agents. Similarly, you could make optimal strategies tractable (a computational problem) by designing mechanisms with a simple truthrevealing equilibrium (an economic solution). However, we will not discuss the other side of the interplay between MD and computer systems-namely, using computing techniques to discover good mechanisms. ${ }^{7,8}$

\section{MD theory}

MD considers a setting with a set of agents $N$, each holding private information $\theta_{i}$ about its preferences $\left(\theta_{i}\right.$ is also known as the type of the agent $i$ ). The type describes how each agent values all possible outcomes, and it is drawn from each agent's available type set $\Theta_{i}$. Agent $i$ with type $\theta_{i}$ has utility $u_{i}\left(\theta_{i}, o\right)$ for outcome $o \in \vartheta$, where $\vartheta$ is the set of possible outcomes. We let the outcome define payments, in addition to decisions in the world, such as task or resource allocations. A mechanism is then a tuple $M=(\Sigma, g)$ that comprises a strategy space (the set of possible actions) $\Sigma$ and an outcome rule $g(\sigma) \in \vartheta$ for $\sigma=\left(\sigma_{1}\right.$, $\left.\sigma_{2}, \ldots \sigma_{|N|}\right) \in \Sigma^{|N|}$. A strategy $s_{i}\left(\theta_{i}\right) \in \Sigma$ defines the actions an agent selects in the mechanism for all possible types $\theta_{i}$. The outcome rule takes the actions agents selected and implements a particular outcome. The mechanism, together with the agent types, defines a game. We assume that agents are autonomous and economically rational and that they select a best-response strategy to maximize their expected utility in equilibrium with other agents. This means no agent can benefit from any unilateral deviation. We assume the mechanism can commit to the outcome rule and implement an outcome.

\section{Mechanism desiderata}

The goal in traditional MD is to design a system in which rational agents interact in a way that leads to equilibriums with desired systemwide properties. These properties are encapsulated in the social choice function (SCF) $f: \Theta \rightarrow \vartheta$, which defines a desired outcome for each possible set of agent types. For example, we might wish to achieve efficiency in the system by maximizing the total utility gained across all agents, in which case

$$
f(\theta)=\max _{o \in \vartheta} \sum_{i \in N} u_{i}\left(\theta_{i}, o\right)
$$

You can imagine allocating computational resources to maximize agent utility or dividing tasks to minimize the system's total cost. In short, the SCF describes the properties that the designer would like MAS outcomes to possess. Given this, the designer's problem is to provide incentives so that agents choose to act in a way that implements a particular SCF. Here are some typical desired properties (desiderata) of SCFs:

- Pareto optimality: Implementing an outcome that is not Pareto-dominated by any other outcome, so no other outcomes make one agent better-off while making other agents worse-off.
- Maximized social welfare: Implementing an outcome that maximizes the total utility across agents. This is often called the efficient outcome.

- Maximized utility to a particular agent: Maximizing the expected utility to a single agent, typically the center, across all possible mechanisms. A common setting is a revenue-maximizing auction, in which the goal is to design a mechanism that maximizes the auctioneer's revenue.

- Budget balance: The total payment that agents make equals exactly zero (a strict budget balance), so money isn't injected into or removed from a system. Or, the total payment is nonnegative (a weak budget balance), so the mechanism doesn't run at a loss. We can also consider an $e x$ ante budget balance, in which the mechanism is balanced on average, and an $e x$ post budget balance, in which the mechanism is balanced at all times, for all instances.

- Individual rationality: The SCF gives each agent nonnegative utility in equilibrium. We can consider interim individual rationality, in which an agent has nonnegative utility in expectation given its own type, and ex post individual rationality, in which an agent always has nonnegative utility.

Budget balance is especially important in systems that must be self-sustaining and require no external benefactor to input money or central authority to collect payments. Yet, budget balance often conflicts with other desiderata, such as efficiency (we return to this later). Individual rationality is important when you can't force agents to participate in a mechanism.

\section{Equilibrium solution concepts}

We would like a mechanism $M=(\Sigma, g)$ to implement SCF $f$ in equilibrium. We can use several different solution concepts in MD to predict the strategies agents will select. Each solution concept differs in assumptions about agents' rationality and about the knowledge that agents have about the system's other agents.

We present the three most useful solution concepts, each successively requiring stronger assumptions about agents (see the "Auctions Illustrating Equilibrium Solution Concepts" sidebar for example auctions).

Dominant strategy. Each agent has a bestresponse strategy no matter what strategy the 


\section{fuctions Illustrating Equilibrium Solution Concepts}

An example of a dominant-strategy implementation is the second-price (Vickrey) auction, where the auction clears for the second-highest price. In this case, the dominant strategy is for an agent to truthfully reveal its valuation for the item.

An example of an ex post Nash implementation is the English auction, an ascending-price auction in which the ask price is $\varepsilon$ above the current winning bid. ${ }^{1} A$ straightforward bidding strategy is to bid at the ask price $p$ whenever $p \leq v_{i}$ for value $v_{i}$. This is an ex post Nash equilibrium - that is, as long as other agents are also straightforward, an agent can do no better, whatever the other agents' values. However, straightforward bidding is not a dominant strategy equilibrium. Consider another agent that conditions a "crazy" strategy such as "I will bid to $\$ 1$ million" if the price hits a particular target value. In this case, an agent should submit a jump bid past this target price to prevent this strategy from triggering. Preventing jump bids in the English auction makes straightforward bidding a dominant strategy.

An example of a Bayesian-Nash implementation is the firstprice sealed-bid auction. Given a symmetric equilibrium of agent types with values that are identically and independently distributed, $v_{i} \sim U(0,1)$, the symmetric BNE is for agents to play $s_{i}^{*}\left(v_{i}\right)=(|N|-1) v_{i} /|N|$.

All of these auctions implement the efficient allocation and are revenue-equivalent in equilibrium.

\section{Reference}

1. R.P McAfee and J. McMillan, "Auctions and Bidding," J. Economics Literature, vol. 25, 1987, pp. 699-738. other agents select. Formally, we have

$$
\begin{aligned}
& s_{i}^{*}\left(\theta_{i}\right)= \\
& \underset{s_{i}}{\arg \max } u_{i}\left(\theta_{i}, g\left(s_{i}\left(\theta_{i}\right), s_{-i}\left(\theta_{-i}\right)\right)\right) \\
& \forall s_{-i}, \forall \theta_{-i}
\end{aligned}
$$

for all $\theta_{i} \in \Theta_{i}$.

A dominant-strategy equilibrium provides a robust solution concept because an agent doesn't need to form beliefs about either other agents' rationality or the distribution over the other agent types.

Ex post Nash. Each agent's strategy is a best response to other agents' strategies no matter what their types, as long as they also play an equilibrium strategy:

$$
\begin{aligned}
& s_{i}^{*}\left(\theta_{i}\right)= \\
& \arg \max _{s_{i}} u_{i}\left(\theta_{i}, g\left(s_{i}\left(\theta_{i}\right), s_{-i}^{*}\left(\theta_{-i}\right)\right)\right) \forall \theta_{-i}
\end{aligned}
$$

for all $\theta_{i} \in \Theta_{i}$, where $s_{-i}^{*}\left(\theta_{-i}\right)$ denotes the equilibrium strategies played by other agents.

An ex post Nash equilibrium requires common knowledge about the agents' rationality but doesn't require any knowledge about type distributions. In this sense, ex post Nash has a no-regret property, and an agent doesn't want to deviate from its strategy even once it knows the other agents' types.

Bayesian-Nash. Each agent selects a bestresponse strategy to maximize its expected utility given its beliefs about the distribution over types, as long as the other agents also play an equilibrium strategy:

$$
\begin{aligned}
& s_{i}^{*}\left(\theta_{i}\right)= \\
& \underset{s_{i}}{\arg \max _{\theta_{-i}}}\left[u_{i}\left(\theta_{i}, g\left(s_{i}\left(\theta_{i}\right), s_{-i}^{*}\left(\theta_{-i}\right)\right)\right)\right]
\end{aligned}
$$

for all $\theta_{i} \in \Theta_{i}$.

The Bayesian-Nash equilibrium is the weakest solution concept adopted in MD. In a BNE, every agent must hold both beliefs about other agents' rationality and correct beliefs about the distribution on types of other agents.

\section{Incentive-compatible mechanisms}

Given solution concepts and SCF desiderata, the central question in MD is, which set of desiderata can be implemented in a MAS's game-theoretic equilibrium, given that the agents are assumed to be self-interested? In this context, the revelation principle is a key concept when it comes to generating impossibility and possibility results. ${ }^{9}$ The revelation principle (see the related sidebar) states that any mechanism can be transformed into an incentive-compatible (IC), direct-revelation mechanism (DRM). In this context, "direct" means the agents' strategy space is restricted to reporting their types and "incentive compatible" means the equilibrium strategy for agents is truth-telling. In the special case of a DRM in which truth revelation is a dominant strategy, we say the mechanism is strategyproof.

The revelation principle is important in MD for two reasons:
- Theoretical: It allows a focus on IC DRMs for the development of impossibility and possibility results.

- Practical: The properties that an IC DRM can satisfy can provide a normative guide for the outcome and payments that a realized implementation must compute. This mechanism need not itself be a DRM and can have better computational properties than the original mechanism.

A central possibility result from an analysis of IC DRMs is the celebrated VickreyClarke-Groves mechanism, a strategyproof mechanism that maximizes the social welfare- - that is, selects the outcome that maximizes the total utility across all agents.

Consider partitioning the outcome space into a choice $k$ and payments $t$. Outcome $o=$ $(k, t)$ defines a choice $k \in \mathbf{K}$ in the space of feasible choices $\mathbf{K}$ and payments $t=\left(t_{1}, \ldots, t_{N}\right)$ by agents. For instance, the choice set can describe all feasible resource allocations to agents. As is common in most auction theory, we assume quasilinear utility functions with

$$
u_{i}\left(k, t_{i}, \theta_{i}\right)=v_{i}\left(k, \theta_{i}\right)-t_{i},
$$

where $v_{i}\left(k, \theta_{i}\right)$ defines the value of allocation $k$ to agent $i$ given its type $\theta_{i}$. The VCG mechanism receives claims $\hat{\theta}_{i}$ from agents about their valuations and implements the choice $k^{*}$ that maximizes $\Sigma_{i} v_{i}\left(k, \hat{\theta}_{i}\right)$. Each agent makes payment $v_{i}\left(k, \hat{\theta}_{i}\right)-(V(N)-V(N \backslash i))$, where $V(N)$ is the total reported value of $k^{*}$ and $V(N \backslash i)$ is the total reported value of the choice that would be implemented without agent $i$. The first two terms of the payment align an agent's incen- 
The intuition behind this principle is simply a reduction argument. Take some arbitrary mechanism $M^{\prime}$ with equilibrium $s^{*}$. Construct a new direct mechanism $M$, which asks agents to report their types and then internally simulates equilibrium $s^{*}$ in mechanism $M^{\prime}$. Given that $s^{*}$ is an equilibrium of $M^{\prime}$, then truth-revelation is an equilibrium of $M$. So, $M^{\prime}$ is incentive compatible.

For example, consider the direct-revelation equivalent of the
English auction, in which straightforward bidding is an ex post Nash equilibrium. The direct-revelation implementation of this asks agents to reveal their valuations and then simulates the English auction with these straightforward bidding strategies on the basis of the revealed valuations. The effect is to sell the item to the agent with the highest bid for the second-highest bid, which is the well-known Vickrey auction. Truthful bidding is a dominant strategy in the Vickrey auction. tives with that of the mechanism and make truth-revelation a dominant strategy. In equilibrium, each agent receives as utility the marginal value that it contributes to the system.

The VCG mechanism is not budget balanced, although it is weakly budget balanced in some settings (for example, in the combinatorial-auction problem that we consider later). In fact, one important impossibility result, the Myerson-Satterthwaite theorem, ${ }^{9}$ shows that no efficient and balanced mechanism can exist in many simple settings, including a simple-exchange setting with a single buyer and a single seller and uncertainty about whether the efficient outcome is to transfer the item from the seller to the buyer. So, in general, if we choose to retain budget balance, we must accept some efficiency loss. Approaches to addressing the budget-balance problem include adjusting payments to get close to VCG payments but retain budget balance, ${ }^{10}$ and retaining truthfulness but explicitly clearing exchanges suboptimally to sacrifice some efficiency in return for budget balance. ${ }^{11}$

However, reiterating the point on practicality, the revelation principle doesn't imply that direct mechanisms are the only ones that are interesting in practice. What the revelation principle does provide is a normative guide for implementing SCFs with particular properties in any particular mechanism realization. In fact, theoretically applying the revelation principle ignores all computational considerations. We see that all equilibrium and outcome calculations are moved to the center, and we assume the agents can report their complete types. So, any indirect mechanism (for example, an ascending-price auction) that implements an efficient allocation in equilibrium must also implement the payments defined in a VCG mechanism. ${ }^{12}$

Designing MASs for self-interested agents involves certain computational considerations. Centralized CMD, like traditional MD, as- sumes a centralized solution. We must also resolve subtle interactions between desirable computational and economic properties that arise when considering the implementation of a particular mechanism in a MAS. While addressing these computational considerations, we should consider the proposed solutions' effect on the system's economic properties.

\section{CMD}

The combinatorial-auction problem (CAP) illustrates the need for CMD and helps identify the challenges. We discuss some methods to address these challenges in the context of combinatorial auctions, which are popular mechanisms for CAP.

\section{Combinatorial auctions}

A combinatorial auction helps determine efficient allocations in settings with multiple items and agents that wish to express complements and substitutes across items (for example, "I only want A if I can also get B" or "I only want AB or CD"). Researchers have proposed combinatorial auctions for many settings, including wireless-spectrumrights allocation, takeoff and landing slots at airports, and multiagent planning. ${ }^{13}$

If the goal is allocative efficiency, then the VCG mechanism provides an economic solution to the CAP. The agents must submit bids on all item combinations (instead of only on items). Then the center solves a winner-determination problem to determine the allocation that maximizes the reported value given agent bids. Buyers must then pay their bid prices for the bundles they receive in the efficient allocation, minus the Vickrey discount. (This is simply the difference between the total value computed by the winner-determination problem with all bidders and the total value computed by the winner-determination problem without that buyer.)

However, the VCG mechanism for the CAP has several undesirable computational characteristics. The winner-determination problem in a combinatorial auction is NPhard and difficult to approximate (being equivalent to the weighted set-packing problem $\left.{ }^{13}\right)$. Furthermore, it's totally centralized, with all agents reporting their complete and exact valuations to the center and the centersolving $|N|+1$ winner-determination problems to determine the allocation and payments. As such, it provides a good canonical example for CMD.

\section{CMD challenges}

Previously, we highlighted key issues concerned with moving from MD to CMD. Of these, the most important challenge relates to bounded computational power, which presents a problem at both the agent level (preference formulation and strategy selection) and the mechanism level (outcome determination). Let's consider each challenge in turn.

\section{Preference formulation and communica-} tion complexity. Naively, we can ask an agent to list all possible outcomes and provide values according to its type. In simple settings, such as voting for candidates in an election, this is easy and doesn't require much computation. However, in more complex settings where the agent must decide between bundles of items or formulate its preferences according to multiple criteria, the problem becomes harder. (This occurs commonly when agents' owners don't specify preference relationships over all outcomes. Rather, they specify the criteria by which to rate these outcomes-for example, specifying preferences for make, color, and price range on cars rather than for all available cars.) The problem has two components. First, an agent must determine its values. Second, an agent must report its values.

One approach proposed to address the first component is to consider indirect mechanisms, such as $i$ Bundle, ${ }^{12}$ that let an agent 
participate in a mechanism without computing or reporting its complete valuation function. Instead, $i$ Bundle is an ascending-price combinatorial auction in which the agent must compute only its best-response bundle set given prices in each auction round. Another approach is more explicit and aims to formulate queries about agent valuations, bypassing the need for agents to formulate preferences for all outcomes. ${ }^{14}$

You can mitigate the second problem component by carefully designing bidding languages to let agents communicate their valuations compactly and expressively. This is important because, for example, a naive-flat bidding language in the CAP would require an agent to specify $2^{|N|}-1$ bids. However, in many cases a bidder might be interested only in a subset of the combinations and might also have a valuation function with useful structure.

Numerous languages, typically based on logical representations, exist for this purpose. ${ }^{15}$ Generally speaking, the literature compares the expressiveness and compactness across families of languages. In the context of indirect mechanisms, such as ascending-price auctions, we also need languages to let agents provide partial information about their preferences-for example, "my value for bundle $S_{1}$ is at least my value for bundle $S_{2}$."12

Strategy selection. An agent must compute its equilibrium strategy given its information about the mechanism and the other agents in the mechanism. If the agent knows a priori that it has a dominant strategy, then the computational problem is insignificant. However, without this knowledge, the agent must compute an equilibrium strategy. Computing the Nash equilibrium in a game is notoriously difficult. It becomes more difficult in an MD context because we are often interested in a Bayesian-Nash equilibrium across games in which agents, owing to their continuous types, can play an infinite number of strategies. ${ }^{16,17}$ (Even in the tranquil setting of complete-information matrix-form games, the complexity of computing a Nash equilibrium for a two-player, general-sum game-a game in which a win-win and lose-lose situation could arise-is unknown but suspected to be not in P.)

We can mitigate the strategy selection problem by designing IC mechanisms and, in particular, using a dominant-strategy implementation. Other approaches taken in CMD have included designing mechanisms that don't require much computation from the agents or developing mechanisms based on models of computationally limited agents. In the former approach, we could design dominant-strategy-based mechanisms because they require minimal agent computation. ${ }^{18}$ However, as we observed earlier, the range of desiderata we can incorporate into mechanisms with dominant strategies is limited. The $i$ Bundle mechanism demonstrates the latter approach. ${ }^{12}$ It adopts an iterative approach in which agents play a myopic best-response strategy - meaning they only consider the outcomes in a limited time window-and only reason about one stage of the game at a time.

Outcome determination. The computational burden in DRMs lies with the mechanism. From the agents' reported valuations, the center must compute the SCF's outcome. Dependent on the SCF, the computation could involve solving an NP-hard combinatorial optimization problem, such as the winner determination problem in the CAP, where the center must compute the allocation of items with the highest total value. The outcome determination problem in mechanisms is heavily researched and has probably been investigated most in the form of the winner determination problem in combinatorial auctions. In this area, three main approaches exist.

The first is to identify and exploit structure. Researchers have identified polynomially solvable cases of the winner determination problem. ${ }^{13,15}$ We could use these cases to impose a bid placement structure. However, this leads to inefficient outcomes if the optimal outcomes require bidding that can't be represented with the imposed structure. Alternatively, we could use the algorithms for polynomially solvable cases only when such a structure is detected in the placed bids. ${ }^{19}$ However, this structure doesn't always occur.

The second approach is to use approximations. Because finding an optimal outcome is NP-hard, this approach attempts to find good outcomes that are close to the optimal but much easier to compute. ${ }^{17}$ The main challenge is to introduce approximations without breaking a mechanism's incentive properties. For example, we can replace the winner determination algorithm in a VCG mechanism with an approximate one and retain strategyproofness, but only if the algorithm satisfies a property of maximal-in-range such that it makes the right decision across a fixed range of choices independent of the valuations that agents report. ${ }^{17}$ (Intuitively, if we're not careful in setting the VCG mechanism, we leave incentives for agents to try to mend the approximations the mechanism introduces through complex game-theoretic reasoning about what other agents will report. This complicates strategy selection.) The literature shows a handful of positive resultsfor instance, for the case of single-minded bidders that desire at most one bundle ${ }^{20}$ and for the case of multiunit allocation problems for a slightly relaxed solution concept. ${ }^{21}$

The third approach is to use an indirect approach. Here, agents interactively compute a mechanism's outcome. For example, they might provide incremental information about preferences or refine that information in response to aggregate signals (via prices, for example) from the mechanism. The basic idea is to share the computational burden between the agent and the mechanism. So, the key questions are

- What kind of strategy space should be designed for the agents if the agents can't just reveal their types?

- How much of the burden should shift to the agents?

- Should we assume that all agents have the same computational resources?

- Can we trust the agents to carry out their share of the computational work?

For example, in $i$ Bundle, agents following straightforward strategies will submit bids for bundles at the current auction prices. ${ }^{12}$ In submitting these bids, the agents are doing some computation (for example, determining bundles with maximal payoff at the current prices). They're also helping the auctioneer by restricting its winner determination to finding the revenue-maximizing allocation solution across the current bids submitted by agents and not in terms of agent's complete valuation functions.

\section{DMD}

So far, we've focused on systems with centers for collecting information from agents and determining and implementing an outcome, and a closed set of agents. We've also assumed in CMD that the agents have a direct, trusted method to communicate with the center, and that they all can report their actions to the center simultaneously. However, these assumptions don't always hold in open distributed systems. For example, consider grid computing, in which a set of resources is distributed over wide-area net- 


\section{Using Mechanism Design to Promote Truth-Telling in PęP Networks}

In current P2P networks such as Gnutella and KaZaa, "free riding"-agents using the system without providing resources toward it-is well-documented. ${ }^{1}$ In response, one approach has been to study the economics of tit-for-tat, where agents can only receive resources to the degree that they contribute them. ${ }^{2}$ However,this approach is blind to the heterogeneity of local agents that will likely differ in their computational resources and data content and quality. Simply stated, freeriding is not necessarily a bad outcome when performed by agents with little to contribute to the network. A mechanism design approach would instead seek to provide incentives for agents to truthfully report local resources (for example, files available for upload, bandwidth speed, and so on) and compute socially-efficient file-sharing solutions.

\section{References}

1. E. Adar and B. Huberman, "Free Riding on Gnutella," First Monday, vol. 5, no. 10, Oct. 2000.

2. K. Lai et al., "Incentive for Cooperation in Peer-to-Peer Networks," Proc. 1st Workshop Economics of Peer-to-Peer Systems, 2003; www. sims.berkeley.edu/research/conferences/p2pecon. works that can support large-scale distributed applications. The Grid is predominantly concerned with coordinated resource sharing and problem solving in dynamic, multiinstitutional, virtual organizations. ${ }^{22}$ As another example, consider P2P systems, which are similar to the Grid but typically have more users with more widely varying capabilities. ${ }^{23}$ Grid and P2P systems have distinct stakeholders and a strong need for effective resource allocation. A need clearly exists for economic design and analysis, but there's no single central agent or controller such as those in classic mechanism design.

At one extreme, we could dispense with MD altogether and simply ask about the "price of anarchy" 24 or the economic cost of just implementing distributed solutions with no carefully designed mechanism. In many cases, we believe that this cost will be too high. So, the challenge remains to design distributed mechanisms that retain normative MD goals (see the sidebar on using MD).

To this end, we explain how to apply DMD while still considering the pertinent problems that must be addressed. In DMD, we consider how to implement an SCF under the constraint that no central agent computes the outcome. On one hand, such a constraint might arise naturally owing to a system's computational structure. On the other hand, such a distributed system has several advantages over centralized MD (which are vital if $\mathrm{MD}$ is to gain a central role in designing MASs with self-interested agents):

- Tractability. A distributed mechanism transfers the computational burden from a central node in the mechanism to the agents. This is like transforming the problem into a distributed optimization problem that exploits many agents' computational resources.
- Robustness. In a centralized mechanism, the communication channels linking to the center are critical for the system, and failure might incapacitate the entire system's operation. However, if these channels fail in a decentralized setting, it won't incapacitate the mechanism, although it could lead to a suboptimal solution.

- Trustworthiness. In a distributed mechanism, because no single agent computes the outcome, a higher degree of trust in the mechanism can exist once you address other incentive issues-why agents will choose to perform their roles appropriately, for example. (Trustworthiness in the center is an ever-present problem in a centralized mechanism, which we believe has influenced the limited use of Internet auctions. For example, in the VCG mechanism we discussed, the auctioneer might proclaim a higher-than-actual second price to increase its revenue.)

- Bottleneck reduction. In distributed mechanisms, communication must no longer pass through a single point.

In DMD, we imagine distributing a mechanism's rules across the agents so that agents are asked, for example, to forward messages following a mechanism-specified protocol or to perform computation on the basis of messages received from other agents following a mechanism-specified protocol. Immediately, we see DMD's intriguing new challenge: the same agents that seek to manipulate a system also run the mechanism. Why, then, wouldn't an agent choose to deviate from implementing a mechanism's proposed rules of the game? So, although trusting a single central agent isn't necessary, we must now have distributed trust - we must trust each agent in a distributed system to implement its piece of the mechanism's outcome.

A distributed Vickrey auction provides a canonical example. Suppose there's a center but no trusted communication channel, and agents must forward a bid from their neighbor toward the center. The only effect that forwarding the bid from its neighbor can have on an agent is to increase competition for the item. In such circumstances, a rational agent would choose to simply drop bids from other agents. One proposed approach to this problem is using redundancy and checker nodes to validate decisions on the basis of revealed information, and partitioning so that either no agent computes outcomes that affect its own utility, or agents must perform computation only when it's in their own interests to be truthful. ${ }^{25}$

Another challenge in developing distributed mechanisms involves reducing the complexity of message passing in the communication network. Consider an interdomain routing problem on the Internet in which individual autonomous systems (or agents) must report their costs for forwarding messages to select the shortest paths between different domains. Incentives matter, but there's no center, and a DMD approach is required to incite agents to reveal truthful information and support shortest-path selection. However, the system should be able to compute this efficient outcome without overburdening the network with messages just to find it. Although not too robust to agents that manipulate the mechanism's implementation, a DMD-based solution is proposed for this problem that only modestly increases the routing-table size and convergence time. ${ }^{26}$

Furthermore, the network topology might in itself affect the type of mechanism we can implement in these systems. ${ }^{25}$ For example, one study has considered the network com- 
plexity of implementing multicast communication on tree-based networks. ${ }^{27}$ This might point to adopting a design methodology similar to that in traditional MD, which develops solutions and mechanisms for restricted domains.

DMD also highlights another issue in applying MD in MASs. MD concentrates almost exclusively on methods to handle rational and self-interested agents' behavior. Yet, most realistic MASs contain various agent behaviors, so this extreme assumption might be too strong. For example, the agents might be obedient (follow the protocol and prescribed strategy), faulty (not function properly or be "irrational" owing to hardware or software failure), strategic (rational, as with the game-theoretic model) or adversarial ("irrational" owing to deviant behavior). ${ }^{28} \mathrm{~A}$ debate already exists about why open P2P systems such as KaZaa work at all given the incentives for users to free-ride. One suggestion is that naive users simply download and run the default client, in which file sharing is enabled. So, in reality we'll need mechanisms based on assumptions about a mixture of agent behaviors, including obedient, strategic, and faulty agents. Traditional equilibrium solution concepts might be inappropriate in these systems with mixed agent behaviors.

Another related problem that arises with both CMD and DMD is that richer kinds of strategic behavior exist than those assumed in most MD theory. Most of MD focuses on the idea that a single agent might unilaterally seek to manipulate a mechanism's outcome. In reality, we might expect agents to collude and form groups that act as one "superagent" to benefit from the reduction in competition and greater power the group wields. However, this behavior might harm the system's overall well-being. (Consider again the Vickrey auction. If the agents having the highest [Agent 1] and second-highest [Agent 2] valuations collude, then these two agents can benefit. For example Agent 1 might offer Agent 2 half the difference between the second-highest and third-highest valuation.) Collusion problems can be more problematic in electronic settings because people may unleash several agents and thus easily adopt multiple identities. Also, they might be bidding across mechanisms, making collusive behavior harder to detect.

\section{Conclusions and general challenges}

In addition to the challenges noted earlier, a number of more general issues cut across both CMD and DMD and still require further investigation.

\section{Online mechanisms}

Many real MASs are dynamic. Rather than taking a decision for a system of agents once, some mechanisms must take a sequence of decisions and maintain a state (for example, a resource allocation) dynamically over time as agents arrive and leave. ${ }^{29}$ For example, consider the problem of Wi-Fi bandwidth allocation in an airport lounge. Clients arrive and leave over time and have heterogeneous valuations for different Wi-Fi connection allocations. An online mechanism is a mechanism that makes a sequence of decisions as the agent types are dynamically revealed as they arrive in the system. The challenge in online MD is to provide appropriate incentive properties so that it's an equilibrium strategy for an agent to both report its true valuation function for different outcomes and announce its arrival as soon as it enters a system.

\section{Adaptive mechanisms}

A second challenge is adaptive-mechanism design, in which the mechanism's rules change over time. Consider the same Wi-Fi bandwidth allocation problem, but where the mechanism doesn't start with a good model of the agents' arrival dynamics or the distribution across agent types. The problem has two components. First, the mechanism must have appropriate incentive properties at any particular instance, even while learning is still taking place and the rules are still suboptimal with respect to some desiderata. Second, the mechanism's dynamics must be robust to withstand manipulation from an agent that wishes to misstate its current type to benefit from this action's dynamic effect on the mechanism's rules later on.

\section{Money}

The presence of money, a common denominator by which every good can be valued, is important in traditional MD. However, in many MASs, such a common denominator doesn't exist naturally and must often be constructed.

\section{Machine-understandable specifications}

Agents act on behalf of their owners. To be effective, owners must communicate their preferences to agents seamlessly. So, the agent's type should basically mirror its owner's.

\section{Semantic interoperation}

Often, numerous institutions provide the same service that an agent requires. So, the agents must be able to operate in all such institutions simultaneously. This should lead to more efficient outcomes because, in this case, the agents will maximize their utility over the whole space of electronic institutions. However, the ability for agents to effectively understand and communicate in an arbitrary institution is a major challenge facing computer scientists.

\section{Regulation and binding contracts}

To prevent a mechanism's incentive properties from unraveling, the center must be able to credibly commit to the game's rules. Similarly, an agent's adherence to the rules laid down by a mechanism can be critical to system functionality. Commitment and trust in the real world are typically built on an intricate set of procedural and legal requirements. This is more difficult in virtual worlds, where legal aspects are less well understood or simply undefined. In MAS systems, additional problems might exist, owing to multiple identities, for example, or because validating the existence (or lack) of particular behaviors can be difficult.

C omputational-mechanism design has an important role to play in developing complex distributed systems comprising multiple interacting agents. It offers a powerful suite of tools for analyzing, predicting, and controlling the behavior of self-interested agents. However, several fundamental scientific questions must still be addressed if this nascent field is to reach its full potential and become a significant paradigm for developing such systems.

\section{Acknowledgments}

Rajdeep K. Dash receives funding from a BAE Systems studentship and an Overseas Research Scholarship. David Parkes gratefully acknowledges support from National Science Foundation grant IIS-0238147.

\section{References}

1. N.R. Jennings, "An Agent-Based Approach for Building Complex Software Systems," Comm. ACM, vol. 44, no. 4, Apr. 2001, pp. 35-41.

2. N.R. Jennings and S. Bussmann, "Agent-Based 
Control Systems," IEEE Control Systems Magazine, vol. 23, no. 3, June 2003, pp. 61-74.

3. M.P. Wellman, "A Market-Oriented Programming Environment and its Application to Distributed Multicommodity Flow Problems," J. Artificial Intelligence Research, vol. 1, Aug. 1993, pp. 1-23.

4. S.H. Clearwater, ed., Market-Based Control: A Paradigm for Distributed Resource Allocation, World Scientific, 1996.

5. J.S. Rosenschein and G. Zlotkin, Rules of Encounter, MIT Press, 1994.

6. T. Sandholm, "Distributed Rational Decision Making," Multiagent Systems: A Modern Approach to Distributed Artificial Intelligence, G. Weiss, ed., MIT Press, 1999, p. 201.

7. V. Conitzer and T. Sandholm, "Complexity of Mechanism Design," Proc. Uncertainty in Artificial Intelligence Conf. (UAI), Morgan Kaufmann, 2002, pp. 103-110.

8. S. Phelps et al., "Co-Evolution of Auction Mechanisms and Trading Strategies: Towards a Novel Approach to Microeconomic Design," Proc. GECCO-02 Workshop Evolutionary Computation in Multi-Agent Systems, Morgan Kaufmann, 2002, pp. 65-72.

9. A. MasColell, M. Whinston, and J.R. Green, Microeconomic Theory, Oxford Univ. Press, 1995.

10. D.C. Parkes, J. Kalagnanam, and M. Eso, "Achieving Budget-Balance with VickreyBased Payment Schemes in Exchanges," Proc. 17th Int'l Joint Conf. Artificial Intelligence (IJCAI 01), Morgan Kaufmann, 2001, pp. 1161-1168.

11. R.P. McAfee, "A Dominant Strategy Double Auction," J. Economic Theory, vol. 56, 1992, pp. 434-450.

12. D.C. Parkes, Iterative Combinatorial Auctions: Achieving Economic and Computational Efficiency, doctoral thesis, Dept. of Computer and Information Science, Univ. of Pennsylvania, 2001

13. S. deVries and R. Vohra, "Combinatorial Auctions: A Survey," INFORMS J. Computing, vol. 15 , no. 3, 2003, pp. 284-309.

14. B. Hudson and T. Sandholm, "Effectiveness of Preference Elicitation in Combinatorial Auctions," Proc. AAMAS-2002 Workshop Agent-Mediated Electronic Commerce (AMEC IV), LNAI 2531, Springer-Verlag 2002, pp. 69-86.

15. N. Nisan, "Bidding and Allocation in Combinatorial Auctions," Proc. ACM Conf. Electronic Commerce, ACM Press, 2000, pp. 1-12.

16. D. Reeves and M.P. Wellman, "Computing Equilibrium in Infinite Games of Incomplete

\section{The A U th 0 is}

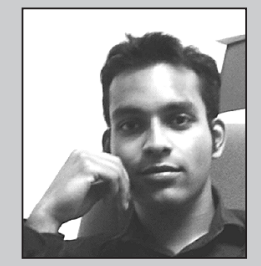

Rajdeep K. Dash is a PhD student in the Intelligence, Agents, Multimedia (IAM) group at the School of Electronics and Computer Science at the University of Southampton. His main interests include distributed-computationalmechanism design, computational-mechanism design, game theory, and multiagent systems. He received his MEng in electrical and electronic engineering from Imperial College. Contact him at IAM Group, Level 4, ECS Dept., Univ. of Southampton, Southampton, SO17 1BJ, UK; rkd02r@ ecs.soton.ac.uk.

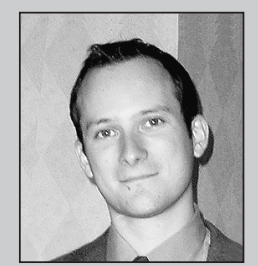

David C. Parkes is a Gordon McKay Assistant Professor of Computer Science at Harvard University. He has published technical papers on e-commerce, auction design, computational-mechanism design, multiagent systems, and bounded rationality. He received his $\mathrm{PhD}$ in computer and information science from the University of Pennsylvania. Contact him at Maxwell Dworkin 229, DEAS, Harvard Univ., Cambridge, MA 02138; parkes@eecs. harvard.edu.

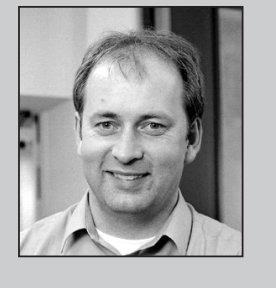

Nicholas R. Jennings is a professor of computer science in the School of Electronics and Computer Science at the University of Southampton. His interests are in the theory and practice of agent-based computing. He received his $\mathrm{PhD}$ from the University of London. He is a fellow of the British Computing Society and the European Coordinating Committee for Artificial Intelligence. Contact him at IAM Group, Level 4, ECS Dept., Univ. of Southampton, Southampton, SO17 1BJ, UK; nrj@ecs.soton.ac.uk.

Information," Proc. AAMAS 03 Workshop on Game-Theoretic and Decision-Theoretic Agents, 2003.

17. A. Ronen, Solving Optimization Problems among Selfish Agents, doctoral thesis, Hebrew Univ., Jerusalem, 2000.

18. H.R. Varian, "Economic Mechanism Design for Computerized Agents," Proc. USENIX Workshop Electronic Commerce, 1995, www. usenix.org/publications/library/proceedings/ ec95/digest.html

19. T. Sandholm and S.Suri, "BOB: Improved Winner Determination in Combinatorial Auctions and Generalizations," Artificial Intelligence J., vol. 145, 2003, pp. 33-58.

20. D. Lehmann, L. Ita O'Callaghan, and Y. Shoham, "Truth Revelation in Approximately Efficient Combinatorial Auctions," J. ACM, vol. 49, no. 5, Sept. 2002, pp. 577-602.

21. A. Kothari, D.C. Parkes, and S. Suri, "Approximately Strategyproof and Tractable Multiunit Auctions," Proc. 4th ACM Conf. Electronic Commerce (EC 03), ACM Press, 2003. pp. 166-175.

22. I. Foster et al., "The Anatomy of the Grid: Enabling Scalable Virtual Organizations," Int'l J. High Performance Computing Applications, vol. 15 , no. 3, Aug. 2001, pp. 200-222.

23. I. Foster and A. Iamnitchi, "On Death, Taxes, and the Convergence of Peer-to-Peer and Grid Computing," Proc. 2nd Int'l Workshop Peerto-Peer Systems, 2003; http://iptps03.cs. berkeley.edu.

24. C.H. Papadimitriou, "Algorithms, Games, and the Internet," Proc. 33rd Ann. ACM Symp. Theory of Computing, ACM Press, 2001, pp. 749-753.

25. J. Shneidman and D.C. Parkes, "Using Redundancy to Improve Robustness of Distributed Mechanism Implementations," Proc. 4th ACM Conf. Electronic Commerce (EC 03), ACM Press, 2003, pp. 276-277.

26. J. Feigenbaum et al., "A BGP-Based Mechanism for Lowest-Cost Routing," Proc. 21st Symp. Principles of Distributed Computing, ACM Press, 2002, pp. 173-182.

27. J. Feigenbaum, C.H. Papadimitriou, and S Shenker, "Sharing the Cost of Multicast Transmissions," J. Computer and System Sciences, vol. 63, no. 1, 2001, pp. 21-41.

28. J. Feigenbaum and S. Shenker, "Distributed Algorithmic Mechanism Design: Recent Results and Future Directions," Proc. 6th Int'l Workshop Discrete Algorithms and Methods for Mobile Computing and Communication, ACM Press, 2002, pp. 1-13.

29. E. Friedman and D.C. Parkes, "Pricing WiFi at Starbucks-Issues in Online Mechanism Design," Proc. 4th ACM Conf. Electronic Commerce, ACM Press, 2003, pp. 240-241. 